\title{
ESTIMATING THE COUNTERPARTY RISK EXPOSURE BY USING THE BROWNIAN MOTION LOCAL TIME
}

\author{
Michele BONOLlO ${ }^{a}$, LUCA DI PERSiO $^{b, *}$, LUCA MAMMI $^{c}$, IMMACOLATA OLIVA $^{d}$ \\ ${ }^{a}$ IMT Lucca/Iason Ltd/Numerix LLC \\ Piazza S. Francesco 19, 55100 Lucca (LU), Via Torino 2, 20123 Milan, Italy \\ e-mail: michele.bonollo@imtlucca.it, mbonollo@numerix.com
}

${ }^{b}$ Department of Computer Science

University of Verona, Strada le Grazie 15, 37134 Verona (VR), Italy

e-mail: luca.dipersio@univr.it

${ }^{c}$ Unicredit Group

Tower A, Piazza Gae Aulenti, 3, 20154 Milan, Italy

e-mail: luca.mammi@unicredit.eu

${ }^{d}$ Department of Economics

University of Verona, Via Cantarane 24, 30129 Verona (VR), Italy

e-mail: immacolata.oliva@univr.it

\begin{abstract}
In recent years, the counterparty credit risk measure, namely the default risk in over-the-counter (OTC) derivatives contracts, has received great attention by banking regulators, specifically within the frameworks of Basel II and Basel III. More explicitly, to obtain the related risk figures, one is first obliged to compute intermediate output functionals related to the mark-to-market position at a given time no exceeding a positive and finite time horizon. The latter implies an enormous amount of computational effort is needed, with related highly time consuming procedures to be carried out, turning out into significant costs. To overcome the latter issue, we propose a smart exploitation of the properties of the (local) time spent by the Brownian motion close to a given value.
\end{abstract}

Keywords: counterparty credit risk, exposure at default, local times Brownian motion, over-the-counter derivatives, Basel financial framework

\section{Introduction}

For some years now, due to the occurrence of events leading to the financial crisis between 2007 and 2008, regulators have forced financial institutions to adopt $a d$ hoc procedures to predict, and therefore prevent, defaults. In other words, banks have to be able to measure and manage their default risk. As for both the credit and the counterparty risk, in 2006 the Basel Committee for Banking Supervision has inserted in the well-known Basel II reform, two rather general methodologies for calculating banks capital requirements, namely the standardized approach and the internal approach. While the former one is based on the use of ratings from external

* Corresponding author credit rating agencies, the latter envisages the evaluation of certain risk parameters, such as the exposure at default (EAD) (cf. BCBS, 2006).

An interesting perspective concerns the so-called counterparty credit risk (CCR), which represents the default risk linked to over-the-counter (OTC) derivatives contracts. The latter case implies the computation, as intermediate outputs, of a large set of different functionals related to the mark-to-market (MtM) of the position over a future time horizon, at a given time $t \in[0, T]$, where $T<+\infty$ is the time horizon. Standard techniques for the evaluation of such an exposure are based on classical Monte Carlo methods, which are characterized by a strong dependence on the number of assets considered 
and related high computational time costs (see, e.g., Liu, 2015). Other approaches have also been given, considering, e.g., a geometric point of view (Sinkala and Nkalashe, 2015), or general ambit stochastic processes (Di Persio and Perin, 2015), or some optimal investment control problems (Chevalier et al., 2013), even if, as a general benchmark, the Monte Carlo set of methods are the most widely used. Nevertheless, as mentioned, Monte Carlo techniques are far from being computationally satisfactory, even in simple cases. For example, a medium bank requires $D=\mathcal{O}\left(10^{4}\right)$ derivative deals and $U=$ $\mathcal{O}\left(10^{3}\right)$ risk factors, evaluated in $K=20$ time steps with $N=2000$ simulations, which allow for $K \times N \times U=$ $4 \times 10^{7}$ grid points for the risk factor simulation and $K \times N \times D=4 \times 10^{8}$ tasks for deals evaluation.

To overcome these drawbacks, the literature has recently proposed new techniques, e.g., vector quantization (Bonollo et al., 2015; Callegaro et al., 2015; 2017; Callegaro and Sagna, 2013), or more enhanced hardware technologies, such as in the case of grid computing and graphical processing units (GPUs) (see, e.g., the works of Castagna (2013) or Pagés and Wilbertz (2011) and the references therein). In the context of American option pricing, other methods recently investigated are the martingale-based approach à la Rogers see, e.g., Lelong (2016), and the simple least-squares approach (see Antonov et al., 2015; Glasserman, 2012). A different solution can be achieved exploiting the so-called polynomial chaos expansion approach (see, e.g., Bernis and Scotti, 2017; Di Persio et al., 2015) and the references therein. Another possibility consists in exploiting the properties of suitable mathematical tools, as for the case of derivatives pricing via Brownian local time. Given a probability space $(\Omega, \mathfrak{F}, \mathbb{P})$, we consider a standard Brownian motion, $\left\{W_{t}\right\}_{t \geq 0}$ defined on it. Then, for $\omega \in \Omega$ and a level $a$, an interesting point is to determine how much time the sample path $W_{t}(\omega)$ spends close to $a$. A possible answer dates back to the works written by Paul Lévy in 1948, where the author introduced the concept of Brownian local time (see Lévy, 1965).

The right approach consists in defining the Brownian local time (BLT from now on) as the following density:

$$
L_{t}(a):=\frac{1}{2 \epsilon} \lim _{\epsilon \longrightarrow 0} \mu\{x:|x-a| \leq \epsilon\},
$$

where $\mu$ represents the Lebesgue measure on the real line.

Remark 1. It is worth mentioning that there does not exist a standard notation to define the BLT, since some authors prefer to multiply the limit in (1) by $1 / 4 \epsilon$, instead of $1 / 2 \epsilon$ (see, e.g., Karatzas and Shreve, 1991).
More formally, the local time can be defined through the so-called occupation formula (see Karatzas and Shreve, 1991), namely by the following equation:

$$
\int_{0}^{t} f\left(W_{s}\right) \mathrm{d} s=2 \int_{\mathbb{R}} f(x) L_{t}(x) \mathrm{d} x,
$$

where the left-hand side is a random measure, called occupation measure or sojourn measure, at fixed time $t$ and level $x \in \mathbb{R}$, while $f$ is an $L^{1}$ function, $f: \mathbb{R} \rightarrow \mathbb{R}$. We refer to Section 3.2 for a more detailed discussion of the BLT properties.

To what concerns the fine properties of the local time, e.g., the identification of both its distribution function and related density function and moments, we refer to the works of Doney and Yor (1998), Karatzas and Shreve (1991) or Takacs (1995), and references therein. It is also worth mentioning that there exist many works dealing with the theoretical applications of the BLT such as an extension of Itô's formula to convex functions, the definition of the density of the occupation measure for a Brownian motion with respect to the Lebesgue measure (Bonollo et al., 2015), etc.

On the other hand, relatively limited literature has been devoted to concrete applications of the BLT and its properties. The latter lack can be easily recognized in frameworks related to economy and finance. Nevertheless, theoretical aspects of the BLT can be fruitfully exploited to analyze a wide range of financial tools, particularly with respect to the pricing of some kinds of exotic path-dependent options as in the case, e.g., of range accrual options and accumulators, where the payoff depends on the time spent by the underlying below or above a given level, between two boundaries, or outside of them (see, e.g., Mijatovic, 2010). Moreover, the use of the BLT is almost absent in the risk management field. The present work aims at filling this gap by showing that the numerical integration of the BLT density function can be used to evaluate the risk exposure, hence obtaining results that are very compelling when compared with classical Monte Carlo benchmark algorithms.

The paper is organized as follows. In Section 2 we introduce the financial framework, focusing on the regulatory viewpoint, and with emphasis to the instructions for calculating the EAD and the credit value adjustment (CVA). Then, in Section 3 the mathematical setting is introduced also recalling the main properties of the BLT, while in Section 4 we provide the local time approach to the aforementioned type of financial problems, also analyzing its performances compared with more standard techniques with respect to an EAD application. Finally, in Section 5 we state our main conclusions and outline future research directions. 


\section{Counterparty risk: The financial framework}

\subsection{Credit counterparty risk in the Basel approach.} In the Basel II framework, the counterparty credit risk (CCR from now on) is a specific class of the broader credit risk category. Let us recall the definition of the Basel committee, shortly BCBS, as it is written by BCBS (2006).

Definition 1. (Counterparty credit risk $(C C R)$ ) is the risk that the counterparty in a transaction could default before the final settlement of the transaction's cash flows. An economic loss would occur if the transactions or portfolio of transactions with the counterparty has a positive economic value at the time of default.

Unlike a firm's exposure to credit risk through a loan, the CCR creates a bilateral risk of loss: the market value of the transaction is uncertain, it can be positive or negative to either counterparty and can vary over time with the movement of the underlying market factors. A typical example is given by IRS. Several classes of financial transactions are considered in the regulatory perimeter, but most of the CCR arise from OTC derivatives, in the peer-to-peer relationships with a defaultable counterparty. From a practical perspective, the buyer of any option, or the holder of a derivative with positive MtM, both are facing a CCR. If the two counterparties agree upon a netting set, e.g., a running compensation process in their deals, the current exposure will be given by the positive part of the algebraic sum of all deals.

As in the whole Basel setting, the risk must be dealt with by setting apart an amount regulatory capital of the bank which is linked to the risk measure called capital requirement (BCBS, 2011):

$$
\begin{aligned}
K= & \operatorname{EAD} \cdot 1.06 \cdot \operatorname{LGD}\left\{\Phi \left[\left(\frac{1}{1-\rho}\right)^{0.5} \Phi^{-1}(\mathrm{PD})\right.\right. \\
& \left.\left.+\left(\frac{\rho}{1-\rho}\right)^{0.5} \Phi^{-1}(0.999)\right]-\mathrm{PD}\right\} \cdot c,
\end{aligned}
$$

where EAD is the exposure at default, namely an estimate of the extent to which a bank may be exposed to a counterparty in the case of a default; LGD is the loss given default, namely an estimate of the percentage of the credit not recoverable in the case of insolvency; PD is the probability of default, namely an estimate of the likelihood that a default will occur; $\rho$ is the asset return correlation coefficient; $c$ is a constant which takes into account some maturity adjustment and may vary with respect to different regulatory portfolios, such as enterprise or retail loans; 1.06 is a coefficient depending on the calibration procedure made by the Basel committee; $\Phi$ is the cumulative distribution function of a standard Gaussian random variable; $\Phi^{-1}$ is simply the inverse of $\Phi$, also referred to as the quantile function.
As well highlighted in the BCBS definition (see Definition 10, the EAD estimate makes the counterparty risk very different from the normal credit risk for loans and mortgages. In fact, the Basel formula (3) requires a one-year measurement process, and the default time $\tau$ could be, or it could not be, AT any future time $t$.

For a mortgage, we know the future exposure profile, since it can be computed using the amortizing plan. Differently, in the CCR, the EAD estimation is fairly difficult, because of two different reasons: the future exposure is stochastic and, further, it depends on the market parameters via its specific evolution pricing model.

In other words, the CCR depends in its magnitude both on the credit parameters (PD, LGD) and on the market influenced EAD parameter; that is why it is also referred to as the boundary risk. To summarize, the CCR has to be determined according to (3) for the credit risk, but its EAD input estimation is itself a hard challenge, to which the Basel committee and the financial operators pay most of their attention.

\subsection{Exposure and CVA calculation in the Basel II-} III setting. In order to calculate the EAD quantity in the CCR context in a robust and conservative way, the Basel II framework (BCBS, 2006) defines two important different approaches: the standard model and the internal model, also called EPE-based approach. In the standard model, we have EAD $=\mathrm{MtM}+$ Add - On, where the Add-On is computed exploiting a table which depends on both the underlying asset class and on the time to maturity. In this case, the idea is that such an Add-On takes into account the future volatility by additive coefficients. As an example, for an equity option with maturity $M$ years and such that $1 \leq M \leq 5$, we have that the Add-On is $8 \%$ of the notional amount, while for an interest rate derivative it is just $0.5 \%$. In the EPE-based approach, to which the present work refers, some notation has to be pointed out. Given a derivative maturity time $0<T<+\infty$, we consider $K \in \mathbb{N}^{+}$time steps $0<t_{1}<t_{2}<\cdots<t_{K}$, which constitute the so-called buckets array, denoted by $\mathbf{B}^{T, K}$, where usually, but not mandatory, $t_{K}=T$. For every $t_{k} \in \mathbf{B}^{T, K}$, we denote by $\operatorname{MtM}\left(t_{k}, S_{k}\right):=\operatorname{MtM}\left(t_{k}, S_{t_{k}}\right)$ the fair value, mark-to-market, of a derivative at time bucket $t_{k}$, with respect to the underlying value $S_{k}$ considered at time $t_{k}$.

For every $t_{k} \in \mathbf{B}^{T, K}$, we denote by $\operatorname{MtM}\left(t_{k}, S^{k}\right):=\operatorname{MtM}\left(t_{k}, S^{t_{k}}\right)$ the fair value (mark-to-market) of a derivative at time bucket $t_{k}$, with respect to the whole sample path $S^{k}:=\left\{S_{t}: 0 \leq t \leq t_{k}\right\}$, and with initial time $t_{0}=0$.

Taking into account previous definitions, we indicate by $\varphi=\varphi\left(T-t_{k}, S_{k}, \Theta\right)$ the pricing function for the given derivative, where $\Theta$ represents the set of parameters from which such a pricing function may depend, e.g., the free risk rate $r$ or the volatility $\sigma$. 
We give an account of the main amounts, as they are defined in Basel III (BCBS, 2006), that will be used later on to estimate the EAD. We introduce the expected exposure of the derivative at time $t_{k} \in \mathbf{B}^{T, K}\left(\mathrm{EE}_{k}\right)$, as

$$
\mathrm{EE}_{k}:=\frac{1}{N} \sum_{n=1}^{N} \operatorname{MtM}\left(t_{k}, S_{k, n}\right)^{+}, \quad N \in \mathbb{N}^{+},
$$

which is the arithmetic mean of the positive part of $N$ Monte Carlo simulated MtM values, computed at the $k$-th time bucket $t_{k}$, with respect to the underlying $\mathrm{S}$.

Remark 2. The positive part operator is effective if we are managing a symmetric derivative, such as an interest rate swap or a portfolio of derivatives. Nevertheless, it is redundant if we consider a single option, as the fair value of the option is always positive from the buy side situation. We want to stress that the sell side does not imply counterparty risk, hence it is out of context.

We evaluate the expected positive exposure (EPE) as

$$
\mathrm{EPE}:=\frac{1}{T} \sum_{k=1}^{K} \mathrm{EE}_{k} \cdot \Delta_{k},
$$

where $\Delta_{k}=t_{k}-t_{k-1}$ indicates the time space between two consecutive time buckets at the $k$-th level. If the time buckets $t_{k}$ are equally spaced, then the formula reduces to $\mathrm{EPE}=\frac{1}{K} \sum_{k=1}^{K} \mathrm{EE}_{k}$. Therefore, the EPE value gives the time average of $\mathrm{EE}_{k}$ and reflects the hypothesis that the default could happen, as a first approximation, at any time with the same probability. We define the effected expected exposure as follows $\mathrm{EEE}_{1}:=\mathrm{EE}_{1} ; \mathrm{EEE}_{k}:=$ $\max \left\{\mathrm{EE}_{k}, \mathrm{EEE}_{k-1}\right\}, k=1, \ldots, K$, observing that, due to its non decreasing property, $\mathrm{EEE}_{k}$ takes into account the fact that, once the time decay effect reduces the MtM as well as the counterparty risk exposure, the bank applies a roll out with some new deals. We also define the effected expected positive exposure (EEPE) by

$$
\mathrm{EEPE}:=\frac{1}{T} \sum_{k=1}^{K} \mathrm{EEE}_{k} \cdot \Delta_{k} .
$$

Remark 3. In order to avoid too many inessential regulatory details, we will work on $\mathrm{EE}_{k}$ and the EPE, the others quantities being just arithmetic modifications of them.

In what follows we shall rewrite previously defined quantities in continuous time, and we add the index $A$ to indicate the adjusted definitions. Moreover we consider the dynamics of the underlying $S_{t}:=\left\{S_{t}\right\}_{t \in[0, T]}, T \in$ $\mathbb{R}^{+}$being some expiration date, as an Itô process, defined on some filtered probability space $\left(\Omega, \mathfrak{F}, \mathfrak{F}_{t \in[0, T]}, \mathbb{P}\right)$. As an example, $S_{t}$ is the solution of the stochastic differential equation defining the geometric Brownian motion, $\mathfrak{F}_{t \in[0, T]}$ being the natural filtration generated by a standard Brownian motion $W_{t}=\left(W_{t}\right)_{t \in[0, T]}$ and with respect to a complete probability space $(\Omega, \mathfrak{F}, \mathbb{P})$, where $\mathbb{P}$ is often referred to as the so-called real world probability measure, or an equivalent risk neutral measure under the martingale approach to option pricing (see, e.g., Karatzas and Shreve, 1991).

The adjusted expected exposure $\mathrm{EE}^{A}$ is given by

$$
\begin{aligned}
\mathrm{EE}_{k}^{A} & :=\mathbb{E}_{\mathbb{P}}\left[\operatorname{MtM}\left(t_{k}, S_{k}\right)^{+}\right] \\
& =\int \varphi\left(T-t_{k}, S_{k}, \Theta\right) \mathrm{dP} \\
& \cong \frac{1}{N} \sum_{n=1}^{N} \operatorname{MtM}\left(t_{k}, S_{k, n}\right)^{+}=\widehat{\mathrm{EE}_{k}^{A}}
\end{aligned}
$$

Similarly, we define the adjusted expected positive exposure $\mathrm{EPE}^{A}$ as

$$
\operatorname{EPE}^{A}:=\int \mathrm{EE}_{t}^{A} \mathrm{~d} t=\iint \varphi\left(t, S_{k}, \Theta\right) \mathrm{d} \mathbb{P} \mathrm{d} t .
$$

With respect to the latter formulation, the Basel definition is simply one of many methods that can be used to estimate the expected fair value of the derivative in the future.

Remark 4. We skip any comment about the choice of the most suitable probability measure $\mathbb{P}$ to be used in the calculation of $\mathrm{EE}_{k}$, the latter being beyond the aim of the present paper. For a detailed discussion on the role played by the risk neutral probability, or by the historical real world probability (see, e.g., Brigo et al., 2013).

Remark 5. Let us underline that the component usually indicated as a discount factor, or a numeraire, is missing in the EPE definition, the latter being a byproduct of the conservative approach used in the risk regulation.

Besides the EAD, understood as a CCR measure, also the credit value adjustment (CVA) may be specified. According to Basel guidelines (BCBS, 2011), the CVA represents the capital charge for potential MtM losses associated with a deterioration in the credit worthiness of a counterparty. Moreover, by introducing the CVA, the expression of the derivative payoff provides a new term, related to the value of the security emerging in the case of a default. In particular, we have

$$
\begin{aligned}
\text { Payoff }= & \phi\left(\mathbf{m}^{T}, \mathbf{c}\right) \cdot \mathbb{1}_{\{\tau>T\}} \\
& +\operatorname{RR} \phi\left(\mathbf{m}^{\mathbf{T}}, \mathbf{c}\right) \cdot \mathbb{1}_{\{\tau \leq T\}},
\end{aligned}
$$

where $\tau$ is the counterparty default time, $\phi\left(\mathbf{m}^{T}, \mathbf{c}\right)$ is the terminal payoff at maturity $T$, where $\mathbf{m}^{T}$, resp. c, stands for the path of the market parameters in $[0, T]$, resp. for the contract clauses on which the payoff depends, while $\mathrm{RR}:=1-\mathrm{LGD}$ is the so called recovery rate, that is, the 
extent to which principal and accrued interest on a debt instrument that is in default can be recovered, expressed as a percentage of the instrument's face value. Hence, the CVA metrics performs an average reduction of the MtM value and involves another form of risk, the CVA risk, characterizing the uncertainty of the future CVA evolution.

Remark 6. Let us note that one of the major credit rating agencies, namely Moody's, estimates defaulted debt recovery rates using market bid prices observed roughly 30 days after the date of default. Recovery rates are measured as the ratio of price to par value (see the report for Moody's (2009) for further details).

2.3. Computational challenges. An extremely interesting and challenging problem consists in the concrete implementation of both the EPE and the CVA. Because of the EPE (EAD) volatility, the counterparty risk must be monitored frequently, hence the standard requirement for an internal model validation is a daily frequency. To have an idea of the magnitude of the computational efforts for such a procedure, let us consider that, in a medium size banking group that aims to satisfy the regulators indications, we could observe $D=10000$ deals in the book, $N=2000$ simulations and $K=20$ time steps. If we denote by PT the number of pricing tasks for each CCR run, we easily get

$$
P T=D \cdot N \cdot K=4 \cdot 10^{8} .
$$

This example easily shows how great the required computational effort is even though a big part of the pricing algorithms is still represented by specific à la Monte Carlo techniques. Hence, although the pricing software and CPU features are adequate for front office purposes, they become unsatisfactory for CCR evaluation constraints. As for the storage requirements, we define the new parameter $\alpha$, i.e., the number of execution cases that have to be stored to allow traceability and auditability of the output results. We can fix $\alpha=13$, if we suppose an end-of-month backup with one-year memory. Of course, the storage is run on different record types, e.g., deal information, payoff information, simulation information, etc. For the sake of simplicity, we can think of the storage as a unique large record type, denoted by RT, which takes into account all the relevant information. Hence

$$
\mathrm{RT}=D \cdot N \cdot K \cdot \alpha=5.2 \cdot 10^{9} .
$$

As each record could easily require 1000 bytes, we raise to 5.2 terabytes of storage. In other words, the CCR involves the computational hard challenges related to the credit and market risk fields. In particular, the high frequency of monitoring implies a number of concrete practical implementations of efficient and robust CCR calculation. In order to address the previous challenges, important results have been achieved exploiting techniques related to the so-called BigData analysis as well as using graphical processing units (GPU); see, e.g., the numerical investigations provided by Castagna (2013) or Pagés and Wilbertz (2011). Nevertheless, the solution to the computational challenges posed by the CCR evaluation are neither completely, nor satisfactory solved by the aforementioned software improvements. That is why there is a growing and wide interest in finding more effective theoretical techniques, and related applied algorithmic procedures.

Remark 7. We would like to underline that while the Basel Committee generally defines frameworks and principles, it does not prescribe a mandatory model or some numerical technique that one has to apply. Hence, starting from the next section, we propose a novel method to perform the EPE calculation, in the broad CCR setting, by exploiting a BLT approach.

\section{Mathematical setting}

3.1. Black-Scholes market model. In what follows we will refer to the celebrated Black and Scholes diffusion process (see Black and Scholes, 1973), as a theoretical benchmark for our proposal's verification. Let us consider a financial market, composed of a risk-less security $B$, with constant return $r$, and a risky asset $S$, defined by means of a geometric Brownian motion, namely

$$
\left\{\begin{array}{l}
\mathrm{d} B_{t}=r B_{t} \mathrm{~d} t, \\
\mathrm{~d} S_{t}=S_{t} \mu \mathrm{d} t+S_{t} \sigma \mathrm{d} W_{t},
\end{array}\right.
$$

where $\mu \in \mathbb{R}, \sigma>0$ and $\left\{W_{t}\right\}_{t>0}$ represents a standard Brownian motion.

The SDE representing the geometric Brownian motion in (10) admits the following unique solution:

$$
S_{t}=S_{0} \exp \left\{\left(\mu-\frac{\sigma^{2}}{2}\right) t+\sigma W_{t}\right\},
$$

which characterizes the dynamics of the underlying of a derivative, namely a financial instrument that gives to its owner a terminal payoff $\phi=\phi\left(\mathbf{m}^{T}, \mathbf{c}\right)$ evaluated at the maturity $T$. To give an example, in the simple case represented by considering a European call option, we have $\phi:=\left(S_{T}-K\right)^{+}$, where the level $K$ is called the strike price of the option, since it provides a positive profit if and only if $S_{T}>K$. Let us recall that the parameters $r$ and $\sigma$ represent the risk-free rate and the volatility of the underlying, respectively. The risk-free rate plays a key role in the evaluation process, that is, the definition of the fair value (FV from now on) at time 0 . In other words, by an application of the Itô-Döblin lemma, it is possible to show that, in the fair value evaluation, 
the actual drift $\mu$, with $\mu>r$, and the unknown risk aversion of the market, or utility function, both disappear, while the fair value can be simply calculated as the discounted expected payoff, where the risk-neutral drift $r$ can straightly replace the expected drift $\mu$ in (11); see, e.g., the work of Hull (1999) for further details. In the basic Black-Scholes simplified model, where the risk-free rate $r$ is deterministic and constant over time, this principle leads to a general evaluation strategy given by

$$
\mathrm{FV}_{t}=\mathrm{E}\left[e^{-r(T-t)} \phi\left(\mathbf{m}^{T}, \mathbf{c}_{t}\right)\right]
$$

The Black-Scholes model gained several extensions and criticism, e.g., sophistication in the payoff algebra, due to the natural innovation process in the financial markets. They allow to cover the effective requirements or to get new profits by issuing new appealing products. Generally speaking, we can have several clauses, e.g., the bundling of different strikes, barriers, memory effects, occupation time clauses, etc., or the dependence of $\phi$ on the whole sample path of $S_{t}$, as it happens when dealing with the so-called Asian, look-back options; new models for the underlying, that arise from the different dynamics among the asset classes, e.g., considering interest rates versus equity versus forex, or from the need of a better calibration of the empirical data, e.g., volatility surface versus flat volatility.

As a benchmark model for an interest rate underlying, the Vasicek model (Vasicek, 1977) and the Hull-White model (Hull and White, 1990) usually replace the Black-Scholes one; an increase in the number of risk sources, e.g., by taking into account the stochastic behavior of volatility, as it happens in the Heston model (Heston, 1993). For a complete review of models, resp. of pricing formulas, see the work of Hull (1999), resp. Haug (1983). Nevertheless, let us recall that, if dealing with a whole portfolio of financial instruments, independently from their features, the Mark-to-Market dynamics can be adequately fitted by a log-normal process because of the compensation or aggregation effect among several single position returns.

This is a common practice in the asset management sector, often referred to as the normal portfolio approach (see, e.g., Saita, 2007). Moreover, also in the risk management approach, the lognormal Black-Scholes model is quite satisfactory, as pointed out, e.g., by Glasserman (2012), who proposes a particular type of incremental risk charge (IRC) model. We recall that, in the real world, one buys or sells a derivative for a given quantity, or notional, namely one takes a position. Hence, in the following, we will often replace the fair value by its related mark-to-market expression (MtM), hence by the fair value equipped with a quantity and a sign.
3.2. Local time and occupation time. Let $\left\{W_{t}\right\}_{t>0}$ be a standard Brownian motion, defined over the probability space $(\Omega, \mathfrak{F}, \mathbb{P})$. The local time for the Brownian motion $W_{t}$, or equivalently, the Brownian local time (BLT), first introduced by Lévy (1965), can be seen as a stochastic process indicating the amount of time spent by the Brownian motion process close to a given level $a \in \mathbb{R}$. To quantify such a random time, Lévy (1965) introduced the following random field:

$$
L_{t}(a)=\frac{1}{2 \varepsilon} \lim _{\varepsilon \rightarrow 0} \mu\left\{0 \leq s \leq t,:\left|W_{s}-a\right| \leq \varepsilon\right\},
$$

where $t \in[0, T], a \in \mathbb{R}$ and $\mu$ is the Lebesgue measure. $L_{t}(a)$ was defined as the mesure de voisinage, and Lévy proved its existence, its finiteness and its continuity, (Lévy, 1965). More rigorously, let us recall the following useful definition:

Definition 2. The random field $\left\{L_{t}(x, \omega):(t, x) \in\right.$ $[0, T] \times \mathbb{R}, \omega \in \Omega\}$ is called a Brownian local time if the random variable $L_{t}(x)$ is $\mathfrak{F}$-measurable, the function $(t, x) \longmapsto L_{t}(x, \omega)$ results to be continuous and

$$
\Gamma_{t}(B, \omega):=\int_{0}^{t} \mathbb{1}_{B}\left(W_{s}\right) \mathrm{d} s=\int_{B} L_{t}(x, \omega) \mathrm{d} x,
$$

with $0 \leq t \leq \infty$ and $B \in \mathcal{B}(\mathbb{R})$.

Let us also recall that the quantity on the left-hand side of 12 is known as the occupation time of the Brownian motion up to time $t$. A crucial theoretical point consists in establishing the BLT existence. This is ensured by Karatzas and Shreve (1991, Thm 6.1.1, Ch. 3). The Brownian local time satisfies several useful properties. For the sake of convenience, we report only the ones that we are going to use for our computational purposes, while we refer the interested reader to Karatzas and Shreve (1991, Section 3.6) for a more comprehensive treatment of the subject as well as for the proofs of the results which we will exploit in what follows.

Proposition 1. For every Borel-measurable function $f$ : $\mathbb{R} \rightarrow[0, T]$, we have

$$
\begin{array}{rl}
\int_{0}^{t} & f\left(W_{s}(\omega)\right) \mathrm{d} s \\
\quad= & \int_{\mathbb{R}} f(x) L_{t}(x, \omega) \mathrm{d} x, \quad 0 \leq t \leq T .
\end{array}
$$

As a consequence of Proposition (1), we have

$$
\int_{0}^{t} \mathbb{1}_{\mathbb{R}}\left(W_{s}\right) \mathrm{d} s=\int_{\mathbb{R}} L_{t}(x, \omega) \mathrm{d} x=t .
$$

The following result is known in the literature as the Tanaka-Meyer decomposition, (see the work of Karatzas and Shreve (1991) for further details). 
Proposition 2. Let us assume that the BLT exists and let $a \in \mathbb{R}$ be a given number. Then the process $\left\{L_{t}(a)\right\}_{0 \leq t \leq T}$ is a nonnegative, continuous, additive functional which satisfies

$$
\begin{aligned}
& L_{t}(a) \\
& \quad=\left(W_{t}-a\right)^{+}-(z-a)^{+}-\int_{0}^{t} \mathbb{1}_{(a,+\infty)}\left(W_{s}\right) \mathrm{d} W_{s},
\end{aligned}
$$

for $0 \leq t \leq T$ and for every $z \in \mathbb{R}$.

Remark 8. Note that the representation given in Proposition 2 can be generalized to a semimartingale.

The Brownian motion spends a random time over any set $A$. Hence it is important to be able to derive its density, namely, the probability that the BLT stands close to a given level $a$, for a time $\mathrm{d} y$. Such a density is given by

$$
g(y ; t, a)=\sqrt{\frac{2}{\pi t}} e^{-\frac{(y+|a|)^{2}}{2 t}},
$$

(see Borodin and Salminen, 2002, Eqn. (1.3.4), p. 155).

\section{Local time proposal for the CCR}

4.1. Application of Brownian local time in finance: Accumulator derivatives. In what follows we focus our attention on a particular type of derivatives, namely the Accumulator, which is a path-dependent forward enhancement without a guaranteed worst case. More precisely, an Accumulator is characterized by a contract, agreed upon two parties, which provides that the investor purchases/sells a pre-determined quantity of stock at a settled strike price $K$, on specified observation days $t_{1}, \ldots, t_{n}, t_{n} \leq T, T$ being the expiry of the contract.

Usually, an Accumulator is linked to an underlying which is an exchange rate, but we have similar payoffs with different names, range accrual, in the broad interest rate derivatives frameworks. An example is given by the FTSE Income Accumulator, identified through the ISIN code XS1000869211, over the FTSE 100 Index, with the plan start date on February 14th, 2014, the plan end date on August 14th, 2020, and the maturity date on August 28th, 2020. The plan is expected to pay every three months, the level depending on how the FTSE 100 Index has performed over the quarter. The maximum income is $6.75 \%$ every year, paid if the underlying closes between 5000 and 8000 points on each weekly observation date. Otherwise, the income will proportionally be reduced, according to the time spent out of the range. Although such a kind of derivative product exhibits some benefits, e.g., a noticeable improvement in the exchange rate, the lack of product costs and the existence of several tailor-made features. On the other hand, there are some drawbacks. The latter allowed the accumulator derivatives to earn the nickname of "I will kill you later" products.

In order to permit more flexibility and to reduce hedging costs, the accumulator contracts may include one or two knock-out barriers in order to restrict the maximum profit and/or the maximum loss by the investor. Basically, if at the end of the $i$-th observation day, the closing price $S_{i}$ of the underlying hits the barrier $H$, for all $i=1, \ldots, n$, then the option stops. We distinguish among accumulator-out one-sided knock-out, accumulator-in one-sided knock-out, accumulator-out range knock-out, accumulator-in range knock-out, depending on whether the investor purchases (resp. sells) a one-sided or range knock-out call (resp. put) and sells (resp. purchases) a one-sided or range knock-out put (resp./ call), with the same strike price, fixing dates and expiry date. Hence, the payoff $\mathscr{P}_{i}$ of an accumulator derivative at the observation day $t_{i}, i=1, \ldots, n$, is given by

$$
\mathscr{P}_{i}= \begin{cases}0 & \text { if } \max _{0 \leq \tau \leq t_{i}} S_{\tau} \geq H, \\ Q\left(S_{t_{i}}-K\right) & \text { if } \max _{0 \leq \tau \leq t_{i}} S_{\tau}<H, S_{\tau} \geq K, \\ g Q\left(S_{t_{i}}-K\right) & \text { if } \max _{0 \leq \tau \leq t_{i}} S_{\tau}<H, S_{\tau}<K,\end{cases}
$$

where $Q$ is the purchase quantity and $g$ is the gearing ratio, both fixed by contract; see, e.g., the work of Lam et al. (2009) for further details. For our purposes, we set $Q=1$ and $g=2$, hence implying that the fair value $F V$ is given by

$$
F V_{i}=\sum_{j=1}^{N}\left[C_{t_{j}}-P_{t_{j}}\right] \cdot e^{-r\left(T-t_{i}\right)},
$$

where $C_{t_{j}}:=C\left(S_{0}, K, T-t_{j}, \sigma, H\right)$, resp. $\quad P_{t_{j}}:=$ $P\left(S_{0}, K, T-t_{j}, \sigma, H\right)$, represents the fair price of a knock-out call option, resp. of knock-out put one. We recall that, by assuming that the underlying evolves according to the Black-Scholes, model, the call price and the put price appearing in 18 have a closed form (see, e.g., Lam et al., 2009).

4.2. Proposal for EE evaluation. In what follows we show how the local time may be used as a handy tool in the evaluation of the counterparty credit risk (CCR) for accumulator derivatives. In the setting described by (10) and (11), it is still possible to determine how long the geometric Brownian motion, remains in the neighborhood of any point $a$, for any given set. In other words, we could attain the density of local time with respect to a geometric Brownian motion; see, e.g., the work of Borodin and Salminen (2002) for further details. In particular, we have

$$
\begin{aligned}
& \mathrm{P}(L(t, a) \in \mathrm{d} y) \\
& \quad=f(y ; t, a, \sigma, \nu, S)
\end{aligned}
$$




$$
\begin{aligned}
= & \sqrt{\frac{2}{\pi t}} \sigma a\left(\frac{a}{S}\right)^{\nu} e^{-\nu^{2} \sigma^{2} \frac{t}{2}-\frac{\left(\sigma^{2} a y+|\log (a / S)|\right)^{2}}{2 \sigma^{2} t}} \\
& +|\nu| \sigma^{2} a\left(\frac{a}{S}\right)^{\nu}\left[e^{-|\nu|\left(\sigma^{2} a y+|\log (a / S)|\right)}\right. \\
& \times \operatorname{Erfc}\left(\frac{\sigma^{2} a y+|\log (a / S)|}{\sigma \sqrt{2 t}}-|\nu| \sigma \sqrt{\frac{t}{2}}\right) \\
& -e^{|\nu|\left(\sigma^{2} a y+|\log (a / S)|\right)} \\
& \left.\times \operatorname{Erfc}\left(\frac{\sigma^{2} a y+|\log (a / S)|}{\sigma \sqrt{2 t}}+|\nu| \sigma \sqrt{\frac{t}{2}}\right)\right],
\end{aligned}
$$

where $t$ represents the time up to which the local time is evaluated, $a$ is the underlying, $\sigma$ is the volatility parameter,

$$
\nu:=-\frac{1}{2}+\frac{r}{\sigma^{2}},
$$

$r$ being the risk-free rate, $S$ represents the spot price, and $\operatorname{Erfc}(z)$ is the complementary error function, namely

$$
\begin{aligned}
& \operatorname{Erfc}(z)=1-\operatorname{Erf}(z), \\
& \operatorname{Erf}(z)=\frac{2}{\sqrt{\pi}} \int_{0}^{z} e^{-x^{2}} \mathrm{~d} x .
\end{aligned}
$$

For convenience, from now on we will not consider the presence of a knock-out barrier.

By recalling the expressions of the payoff and the fair value stated in (17) and (18), and supposing a high fixing frequency, we obtain

$$
\begin{aligned}
\mathcal{P}^{(\mathrm{LT})} & =\sum_{j=1}^{N}\left[\left(S_{t_{j}}-K\right)^{+}-2\left(K-S_{t_{j}}\right)^{+}\right] \\
& \approx \int_{0}^{T}\left[\left(S_{t}-K\right)^{+}-2\left(K-S_{t}\right)^{+}\right] \mathrm{d} t \\
& =\int_{\mathbb{R}} L(T, x)\left[(x-K)^{+}-2(K-x)^{+}\right] \mathrm{d} x
\end{aligned}
$$

where the last equality in (20) follows exploiting (13), while $L(t, x)$ is the BLT up to maturity $T$. As a consequence, we are able to evaluate the corresponding fair value for every observation day $t_{i}, i=1, \ldots, n$,

$$
\begin{aligned}
F V_{t_{i}}^{(\mathrm{LT})} & \\
= & e^{-r\left(T-t_{i}\right)} \mathbb{E}\left(\int _ { \mathbb { R } } L ( T , x ) \left[(x-K)^{+}\right.\right. \\
& \left.\left.\quad-2(K-x)^{+}\right] \mathrm{d} x\right) \\
= & e^{-r\left(T-t_{i}\right)} \int_{\mathbb{R}} \mathbb{E}[L(T, x)]\left[(x-K)^{+}\right. \\
& \left.-2(K-x)^{+}\right] \mathrm{d} x \\
= & e^{-r\left(T-t_{i}\right)} \int_{\mathbb{R}} \int_{0}^{\infty} y f(y ; T, x, \sigma, \nu, S) \\
\quad \times & {\left[(x-K)^{+}-2(K-x)^{+}\right] \mathrm{d} y \mathrm{~d} x, }
\end{aligned}
$$

basically as an application of the Fubini theorem, in the second equality, and by the very definition of the BLT density given in 19 .

Hence, as an intermediate first application, we use the above pricing formula for our Accumulator, and we compare three different pricing techniques for the $A c$ cumulator defined by $(C-2 P)$, where $C$ and $P$ are respectively the Call option price and the Put option price, namely: $\mathrm{BSD}$, the straight $\mathrm{BS}$ evaluation, i.e., Eqn. (18); BSC, the continuous time version of BSD, described in Section 4.3, LT: the time proposal given by the formula (21). For a more detailed discussion of the aforementioned quantities, i.e., concerning BSD, BSC and LT, see Section 4.3. The results have been reported in Table 1 and they have been obtained setting $S_{0}=1$, with $N=250$ fixing dates. We can see that the accuracy is very good, with just a small decay when the volatility parameter increases. We are interested in evaluating the EE and EPE introduced in Section 2.2. Hence, for all $t_{i}, i=1, \ldots, n$, we have

$$
\begin{aligned}
\mathrm{EE}_{t_{i}}^{(\mathrm{LT})}= & \mathbb{E}\left(F V_{t_{i}}^{(\mathrm{LT})}\right) \\
\mathrm{EPE}_{t_{i}}^{(\mathrm{LT})}= & \frac{1}{T} \int_{0}^{T} \int_{\mathbb{R}} e^{-r(T-t)} \mathbb{E}(L(T, x)) \\
& \times\left[(x-K)^{+}-2(K-x)^{+}\right] \mathrm{d} x \mathrm{~d} t .
\end{aligned}
$$

Remark 9. By recalling that the expectation functional $\mathbb{E}$ involves integration, it follows that the EPE requires the evaluation of a triple integral. In consequence, we have two further integration steps with respect to the usual MtM current evaluation of the deal, against the expectation with respect to the market parameters scenarios and the time average, respectively.

Remark 10. We wonder which probability measure is better to use when the expectation functional is evaluated. In other terms, we are interested in choosing the most appropriate distribution at any time $t$ and for all market parameters, which represent the input data for the pricing function. As is well known in the literature, there are two alternatives, namely the risk neutral distribution, or the historical one. Since we mainly focus on computation issues, we believe that the latter is not a relevant point. Anyway, in agreement with the majority of the authors, we follow the convention of adopting the historical distribution. In the Black-Scholes framework, the latter implies that there is a real world drift $\mu$ different from the risk-free rate $r$, and such that $\mu>r$.

4.3. Application and numerical results. To the extent of testing the goodness of our local time proposal to estimate the EE as well as the EPE, we compare the algorithm described in the previous subsection with a benchmark à la Black and Scholes (BSD). First of all, 
Table 1. Comparison between fair values obtained with the three methods.

\begin{tabular}{|c|c|c|c|c|c|c|}
\hline $\mathrm{r}$ & $\mathrm{K}$ & $\sigma$ & FV BSD & FV BSC & FV LT & $\Delta(L T, B S D)$ \\
\hline \hline 0.01 & 0.9 & $15 \%$ & 0.0961 & 0.0961 & 0.0961 & $0.00 \%$ \\
0.01 & 0.9 & $25 \%$ & 0.0783 & 0.0784 & 0.0781 & $-0.26 \%$ \\
0.01 & 1 & $15 \%$ & -0.0323 & -0.0322 & -0.0322 & $-0.31 \%$ \\
0.01 & 1 & $25 \%$ & -0.0587 & -0.0585 & -0.0576 & $-1.87 \%$ \\
0.02 & 0.9 & $15 \%$ & 0.1008 & 0.1008 & 0.1008 & $0,00 \%$ \\
0.02 & 0.9 & $25 \%$ & 0.0837 & 0.0837 & 0.0839 & $0,24 \%$ \\
0.02 & 1 & $15 \%$ & -0.0248 & -0.0247 & -0.0247 & $-0.40 \%$ \\
0.02 & 1 & $25 \%$ & -0.0509 & -0.0508 & -0.0501 & $-1.57 \%$ \\
\hline
\end{tabular}

let us fix the number of simulations, indicating them by Nsim. Then, for every simulation,

- we consider Nday $=250$ business days, indicated by $t_{i}, i=1, \ldots$, Nday, for each of which $t_{i}$, we simulate the price of the underlying by using the following discretization procedure:

$$
S_{t_{i}}=S_{t_{i-1}} e^{\left\{\left(r-\frac{\sigma^{2}}{2}\right) \Delta t_{i}+\sigma \sqrt{\Delta t_{i}} \cdot \mathcal{N}(0,1)\right\}},
$$

where

$$
\Delta t_{i}=t_{i}-t_{i-1}=\frac{1}{\text { Nday }}, \forall i=1, \ldots, \quad \text { Nday } .
$$

- then we compute the Accumulator price by means of the following formula:

$$
\begin{aligned}
& \mathrm{FV}_{t_{i}}^{(\mathrm{BSD})} \\
& \quad=\Delta\left(\sum_{j=1}^{i}\left[\left(S_{j}-K\right)^{+}-2\left(K-S_{j}\right)^{+}\right]\right. \\
& \left.\quad+\sum_{k=i+1}^{\text {Nday }}\left[C_{t_{k}}-2 \cdot P_{t_{k}}\right]\right) \cdot e^{-r\left(T-t_{i}\right)}
\end{aligned}
$$

where

$$
\Delta:=\frac{T}{\text { Nday }}
$$

and

$$
\begin{aligned}
C_{t_{k}} & =C\left(S_{0}, K, r, \sigma, T-t_{k}\right), \\
P_{t_{k}} & =P\left(S_{0}, K, r, \sigma, T-t_{k}\right)
\end{aligned}
$$

are the call and put prices, respectively.

In order to evaluate the counterparty credit risk, we choose 10 time steps, one every 25 business days. Then we determine the expected exposure $\mathrm{EE}^{(\mathrm{BSD})}$ and the expected positive exposure $\mathrm{EPE}^{(\mathrm{BSD})}$ by using (4), and (5). Since an Accumulator derivative is characterized by a daily, at least, fixing frequency, we could take into account a continuous version of the derivative fair value. Hence we consider

$$
\mathrm{FV}_{t}^{(\mathrm{BSC})} \cong \int_{0}^{T} e^{-r(T-t)}\left(C_{t}-2 P_{t}\right) \mathrm{d} t
$$

where

$$
\begin{aligned}
C_{t} & =C\left(S_{0}, K, r, \sigma, T-t\right), \\
P_{t} & =P\left(S_{0}, K, r, \sigma, T-t\right)
\end{aligned}
$$

are the call and put prices computed as before. Equation (25) allows us to consider a continuous version of the benchmark, denoted by BSC. In order to compare the LT and BSC approaches, we carry out a time discretization approximating the BSC by retracing the steps of the BSD algorithm and by considering $10^{4}$ fixing dates, namely 40 observations per day, instead of one. Finally, we are able to appraise the expected exposure $\mathrm{EE}^{(\mathrm{BSC})}$, resp. the expected positive exposure $\mathrm{EPE}^{(\mathrm{BSC})}$, again by exploiting (4), resp., (5). As regards the local time algorithm, we use a numerical integration, and, in order to have such an integration as efficient as possible, we fixed convenient lower and upper bounds.

Numerical results. To show how the local time techniques behave compared with classical approaches, we provide the results reported in Table 2 which contains the EPE values obtained with methods introduced in the previous sections. More precisely, we run all the algorithms for several strike, volatility and risk-free parameters, according to the following choices: spot price $S_{0}=5.7$; strike price: $K=[4.78,3.75,2.98]$; volatility: $\sigma=[0.15,0.2,0.3]$ risk-free rate: $r=[0.01,0.02]$ We have analyzed the aforementioned three methods, whose values are described in columns 2-4, focusing on the changes $(\Delta)$ in the EPE values in columns 5-7. Every row is characterized by a triplet $\left(K_{i}, \sigma_{j}, r_{h}\right), i=$ $1, \ldots, 3, j=1, \ldots, 3, h=1,2$, to specify which values of strike price, volatility and risk-free rate we refer.

Remark 11. Let us underline the meaning of the three $\Delta$ comparisons in the right part of Table 2. $\Delta$ (BSC, BSD) does not take into account our proposal, but it measures the difference between the real world (BSD), where the fixing is discrete over time, and its continuous version, namely the BSC one. $\triangle(\mathrm{BSC}, \mathrm{LT})$ has a double role. On one hand, it measures the rightness of our algorithm implementation, as the two methods are theoretically equivalent. Once we verify that the difference is small, 
Table 2. Expected positive exposure of an accumulator derivative.

\begin{tabular}{|c|c|c|c|c|c|c|}
\hline$(K, \sigma, r)$ & BSD & BSC & LT & $\Delta(B S D, B S C)$ & $\Delta(\mathrm{BSC}, \mathrm{LT})$ & $\Delta(\mathrm{BSD}, \mathrm{LT})$ \\
\hline \hline$(4.78,0.15,0.01)$ & 0.9303454395 & 0.9303714275 & 0.9303781163 & $-0.00279 \%$ & $0.00072 \%$ & $0.00351 \%$ \\
$(4.78,0.2,0.01)$ & 0.9049015095 & 0.9049413280 & 0.9048279190 & $-0.00440 \%$ & $-0.01253 \%$ & $-0.00813 \%$ \\
$(4.78,0.3,0.01)$ & 0.8251642939 & 0.8251928714 & 0.8247838254 & $-0.00346 \%$ & $-0.04957 \%$ & $-0.04611 \%$ \\
$(3.75,0.15,0.01)$ & 1.9686102762 & 1.9686111645 & 1.9686848833 & $-0.00005 \%$ & $0.00374 \%$ & $0.00379 \%$ \\
$(3.75,0.2,0.01)$ & 1.9675941521 & 1.9676004514 & 1.9676336107 & $-0.00032 \%$ & $0.00169 \%$ & $0.00201 \%$ \\
$(3.75,0.3,0.01)$ & 1.9547899122 & 1.9548275248 & 1.9547735333 & $-0.00192 \%$ & $-0.00276 \%$ & $-0.00084 \%$ \\
$(2.98,0.15,0.01)$ & 2.7348505526 & 2.7348507540 & 2.7349168463 & $-0.00001 \%$ & $0.00242 \%$ & $0.00242 \%$ \\
$(2.98,0.2,0.01)$ & 2.7348375084 & 2.7348378657 & 2.7348585689 & $-0.00001 \%$ & $0.00076 \%$ & $0.00077 \%$ \\
$(2.98,0.3,0.01)$ & 2.7336498018 & 2.7336570672 & 2.7336545073 & $-0.00027 \%$ & $-0.00009 \%$ & $0.00017 \%$ \\
$(4.78,0.15,0.02)$ & 0.9556220367 & 0.9556450742 & 0.9558308683 & $-0.00241 \%$ & $0.01944 \%$ & $0.02185 \%$ \\
$(4.78,0.2,0.02)$ & 0.9318141129 & 0.9318494415 & 0.9318254905 & $-0.00379 \%$ & $-0.00257 \%$ & $0.00122 \%$ \\
$(4.78,0.3,0.02)$ & 0.8548444479 & 0.8548671968 & 0.8542861489 & $-0.00266 \%$ & $-0.06797 \%$ & $-0.06531 \%$ \\
$(3.75,0.15,0.02)$ & 1.9871484085 & 1.9871500602 & 1.9873803563 & $-0.00008 \%$ & $0.01159 \%$ & $0.01167 \%$ \\
$(3.75,0.2,0.02)$ & 1.9862637277 & 1.9862700504 & 1.9864841609 & $-0.00032 \%$ & $0.01078 \%$ & $0.01110 \%$ \\
$(3.75,0.3,0.02)$ & 1.9743416072 & 1.9743766907 & 1.9744396571 & $-0.00178 \%$ & $0.00319 \%$ & $0.00497 \%$ \\
$(2.98,0.15,0.02)$ & 2.7496039372 & 2.7496047313 & 2.7497784936 & $-0.00003 \%$ & $0.00632 \%$ & $0.00635 \%$ \\
$(2.98,0.2,0.02)$ & 2.7495932160 & 2.7495941376 & 2.7497625846 & $-0.00003 \%$ & $0.00613 \%$ & $0.00616 \%$ \\
$(2.98,0.3,0.02)$ & 2.7485174039 & 2.7485245347 & 2.748661624 & $-0.00026 \%$ & $0.00499 \%$ & $0.00525 \%$ \\
\hline
\end{tabular}

Table 3. Average elapsed time of the three algorithms, measured in seconds.

\begin{tabular}{|c|c|c|}
\hline BSC & BSD & LT \\
\hline \hline 18.314768 & 4.98831 & 2.12311 \\
\hline
\end{tabular}

with a more practical perspective it allows us to monitor the numerical accuracy of the tools we used to perform the various numerical integration involved in both the techniques. Finally, $\Delta(\mathrm{BSD}, \mathrm{LT})$ considers both the previous effects and measures the global accuracy of our BLT proposal, where we proxy, by continuous time, the real world problem by a new, local time based, technique.

In order to complete the comparison between the different methods proposed, we draw a parallel between the execution times of the individual methods, which is reported in Table 3. In particular, we invite the reader to dwell on the last two columns, for which the computational effort is comparable. We observe that the elapsed time of the local time algorithm is less than the elapsed time of the BSD approach and, on the average, the former is about half the latter. Finally, we exhibit a couple of graphs comparing the errors of the algorithm $\mathrm{LT}$ and $\mathrm{BSC}$, with respect to the exact case BSD, once the strike price and the free risk rate have been set, while the volatility $\sigma$ changes. We observe that the relative error in very good for small volatilities. To this extent, we will investigate further the software implementation details. Anyway, referring to the computational time in the Table 3 above, we think that in the usual trade-off (accuracy, time) the LT approach undoubtedly dominates the BSC approximation, and it can compete with the true BSD model.

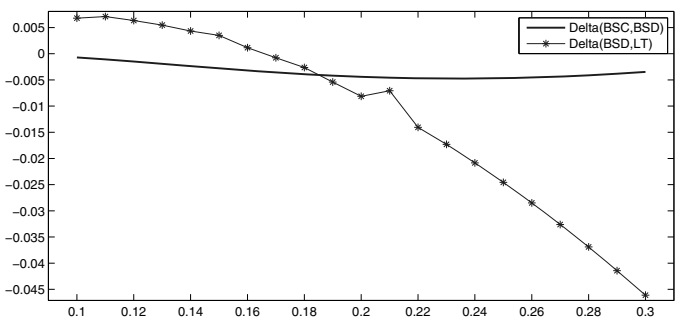

(a)

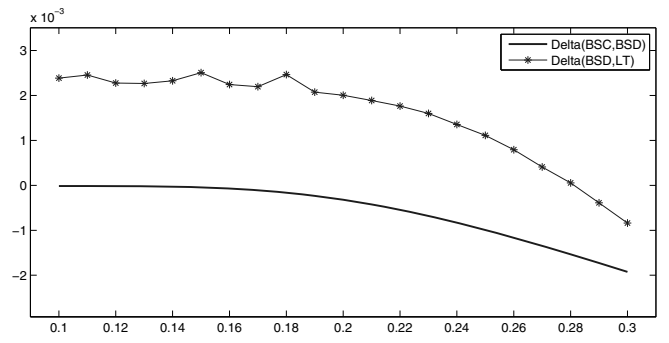

(b)

Fig. 1. Comparison between EPE changes for fixed risk-free rates: $\Delta(\mathrm{BSD}, \mathrm{BSC})$ vs. $\Delta(\mathrm{BSD}, \mathrm{LT})$ with $r=1 \%$ and $K=3.75$ (a), $\Delta(\mathrm{BSD}, \mathrm{BSC})$ vs. $\Delta(\mathrm{BSD}, \mathrm{LT})$ with $r=1 \%$ and $K=4.78$ (b). The volatility parameter varies between $10 \%$ and $30 \%$.

4.4. Some remarks about computational complexity. Once a new methodology or algorithm has been proposed, one would like to make a general analysis of the computation complexity of the new method compared with its more traditional competitors; the some concerns the accuracy and the convergence rate. In the simplest 
and naive case, one has just one parameter, $N$, say, e.g., the number of simulations, the number of deals in the portfolio, the number of time steps, etc., and the computational complexity could be stylized by a single "order" such as $\mathcal{O}(N), \mathcal{O}\left(N^{2}\right)$ and so on. Despite this elegant theoretical approach, concrete applications are characterised by extra difficulties. First, the proposal, or the set of competitors, could depend on some different parameters, and $N$ could not be a proper summary of the technique set up. Second, for each atomic algorithmic task, namely for any simulation of a loop of $N$ simulations, the different competitors could contain calculations with very different levels of complexity and elapsed time, $t_{1}$ and $t_{2}$, say. Hence it may happen that for small or medium values of the parameter $N$ the actual computational time of the two algorithms does not match the asymptotic order ranking, e.g., it may happen that $t_{1} \cdot N>t_{2}$. $N^{3 / 2}$. Third, finally, the observed computational times depend on many implementation details: the numerical integration method, bounded or unbounded integration, the efficiency of the libraries embedded in the exploited programming languages.

Coming back to the above tables of execution times for BSD, BSC and LT, also focusing on the evaluation of the EE and EPE values, loops behave similarly with the cross-method in increasing the number of calculations, and we observe that the BSC involves a time integration of the rather complicated BS formula, while the BSD has a complexity given by $\mathrm{BS} \cdot t_{n}$, the second term being the number of fixing times, and eventually the LT has a complexity given by time-space integration of a quite simple function which is the payoff itself. Moreover, we optimized the latter by bounding both the infimum and the supremum of the space integral. Therefore, even without an exhaustive comparison, also for various implementations, we can conclude that the LT proposal allows for a good Accuracy versus Effort trade-off. We also underline that extensions to other market parameters, clauses and payoffs are needed.

\section{Conclusions and further research}

We have addressed the issue of the CCR assessment for the so-called accumulator derivatives, within the Black-Scholes, financial framework with one risky asset. Since the corresponding payoff depends on the time spent by a geometric Brownian motion near a given value, we have exploited the notion of the BLT which turns to play a crucial role in the derivative pricing step for CCR evaluation. However, it is possible to involve the BLT also in the risk factors simulation step: roughly speaking, for each time bucket $t_{k}$, we could employ the BLT to build up the grid $\left(t_{k}, S_{t_{k}, n}\right)$ and the corresponding probabilities, and evaluate the $k$-th expected exposure $\mathrm{EE}_{k}$ as the sum of weighted probability masses. We have proposed an original approach founded on the possibility of expressing the BLT in terms of its probability density.

The associated implementation with regard to EPE evaluation leads to numerical results that significantly improve those obtained by standard procedures à la Black-Scholes. A smaller execution time and a better EE appraisal accuracy make our method a competitive tool, suggesting an extension of the local time approach to more general derivatives, such as barrier options or Asian options.

The next step consists in comparing our results with those derived by Cordoni and Di Persio (2014; 2016). Moreover, we also plan to use the results presented by Takacs (1995), namely a generalization of the well-known Lévy's arc-sine law; see also Lévy (1939), who provides the distribution of the occupation time given in (12). In fact, we intend to use the related Takacs formula as an alternative expression for the probability density stated in (19) which has been extensively used in this paper.

Finally, we are aware that the one-dimensional case turns out to be unrealistic, though relatively easy to implement, albeit to work in the one-dimensional framework is a very acceptable proxy for derivatives of banks with corporate customers, i.e., small and medium size enterprises; in these cases the $i$-th customer has a very small number of deals, with the main dependence on a single risk factor, e.g., the EUR interest rate curve. After all, a large number of risk factors entails a very hard estimation of correlations.

To overcome such drawbacks, financial institutions resort to some heuristic and easy-to-extend methods. For example, in the two-dimensional case it is common practice to consider

$$
\left\langle\mathrm{d} W_{t}^{(1)}, \mathrm{d} W_{t}^{(2)}\right\rangle=0
$$

between the asset classes, e.g., interest rate, forex or equity, and

$$
\left\langle\mathrm{d} W_{t}^{(1)}, \mathrm{d} W_{t}^{(2)}\right\rangle=\mathrm{d} t
$$

within the asset class. Such a procedure could be easily extended to the $N$-dimensional case, with $N \gg 1$. This is clearly a complicated issue. From a theoretical point of view, the literature provides contributions related to the study of the multidimensional BLT; see, e.g., the work of Brydges et al. (2007) and the references therein.

\section{Acknowledgment}

The first author thanks the Crisis Lab for the financial support. The second author acknowledges the GNAMPA project Set-valued and Optimal Transportation Theory Methods to Model Financial Markets with Transaction Costs Both in Deterministic and Stochastic Frameworks, which has funded the present research. 


\section{References}

Antonov, A., Issakov, S. and Mechkov, S. (2015). Backward induction for future values, Risk.net, Numerix research paper, http://www.risk.net/ derivatives/2387384/backward-inductionfuture-values

BCBS (2006). Basel II: International convergence of capital measurement and capital standards: A revised framework-comprehensive version, Technical Report 128, BCBS Paper, http://www.bis.org /publ/bcbs128.paf

BCBS (2011). Basel III: A global regulatory framework for more resilient banks and banking systems-revised version, Technical Report 189, BCBS Paper, http: //www.bis.org/publ/bcbs189.pdf

Bernis, G. and Scotti, S. (2017). Alternative to beta coefficients in the context of diffusions, Quantitative Finance 17(2): 275-288.

Black, F. and Scholes, M. (1973). The pricing of options and corporate liabilities, The Journal of Political Economy 81(3): 637-654.

Bonollo, M., Di Persio, L., Oliva, I. and Semmoloni, A. (2015). A quantization approach to the counterparty credit exposure estimation, http://ssrn.com/ abstract $=2574384$

Borodin, A. and Salminen, P. (2002). Handbook of Brownian Motion: Facts and Formulae, 2nd Edn., Birkhäuser, Basel.

Brigo, D., Morini, M. and Pallavicini, A. (2013). Counterparty Credit Risk, Collateral and Funding: With Pricing Cases for All Asset Classes, Wiley, Chichester.

Brydges, D., Van Der Hofstad, R. and Konig, W. (2007). Joint density for the local times of continuous-time Markov chains, The Annals of Probability 35(4): 1307-1332.

Callegaro, G., Fiorin, L. and Grasselli, M. (2015). Quantized calibration in local volatility, Risk.net (Cutting Edge: Derivatives Pricing) (2015): 56-67.

Callegaro, G., Fiorin, L. and Grasselli, M. (2017). Pricing via quantization in stochastic volatility models, Quantitative Finance, DOI: 10.1080/14697688.2016.1255348, (in print).

Callegaro, G. and Sagna, A. (2013). An application to credit risk of a hybrid Monte Carlo optimal quantization method, Journal of Computational Finance 16(4): 123-156.

Castagna, A. (2013). Fast computing in the CCR and CVA measurement, Technical report, IASON Working paper.

Chevalier, E., Ly Vath, V. and Scotti, S. (2013). An optimal dividend and investment control problem under debt constraints, Journal on Financial Mathematics 4(1): 297-326.

Cordoni, F. and Di Persio, L. (2014). Backward stochastic differential equations approach to hedging, option pricing and insurance problems, International Journal of Stochastic Analysis 2014, Article ID: 152389, DOI: $10.1155 / 2014 / 152389$
Cordoni, F. and Di Persio, L. (2016). A BSDE with delayed generator approach to pricing under counterparty risk and collateralization, International Journal of Stochastic Analysis 2016, Article ID: 1059303, DOI: 10.1155/2016/1059303.

Di Persio, L., Pellegrini, G. and Bonollo, M. (2015). Polynomial chaos expansion approach to interest rate models, Journal of Probability and Statistics 2015, Article ID: 369053, DOI: $10.1155 / 2015 / 369053$.

Di Persio, L. and Perin, I. (2015). An ambit stochastic approach to pricing electricity forward contracts: The case of the German energy market, Journal of Probability and Statistics 2015, Article ID: 626020, DOI: 10.1155/2015/626020.

Doney, R. and Yor, M. (1998). On a formula of Takacs for Brownian motion with drift, Journal of Applied Probability 35(2): 272-280.

Glasserman, P. (2012). Risk horizon and rebalancing horizon in portfolio risk measurement, Mathematical Finance 22(2): 215-249.

Haug, E. (1983). The Complete Guide to Option Pricing Formulas, McGraw-Hill, New York, NY.

Heston, S. (1993). A closed-form solution for options with stochastic volatility with applications to bond and currency options, The Review of Financial Studies 6(2): 327-343.

Hull, J. (1999). Options, Futures, and Other Derivatives, Pearson Education, Englewood Cliffs, NJ.

Hull, J. and White, A. (1990). Pricing interest-rate derivative securities, The Review of Financial Studies 3(4): 573-592.

Karatzas, I. and Shreve, S. (1991). Brownian Motion and Stochastic Calculus, Springer-Verlag, New York, NY.

Lam, K., Yu, P. and Xin, L. (2009). Accumulator pricing, 2009 Symposium on Computational Intelligence for Financial Engineering, Nashville, TN, USA.

Lelong, J. (2016). Pricing American options using martingale bases, https://hal.archives - ouvertes.fr/hal-01299819.

Lévy, P. (1939). Sur certains processus stochastiques homogènes, Compositio Mathematica 7: 283-339.

Lévy, P. (1965). Processus stochastiques et mouvement brownien, Gauthier-Villars, Paris.

Liu, Q. (2015). Calculation of credit valuation adjustment based on least square Monte Carlo methods, Mathematical Problems in Engineering 2015, Article ID: 959312, DOI: 10.1155/2015/959312.

Mijatovic, A. (2010). Local time and the pricing of time-dependent barrier options, Finance and Stochastics 14(13): 13-48.

Moody's (2009). Moody's global corporate finance recovery rates, Technical report, Moody's Investors Service.

Pagés, G. and Wilbertz, B. (2011). GPGPUs in computational finance: Massive-parallel computing for American style options, Technical report, Laboratoire de Probabilité, Paris VI-VII. 
Saita, F. (2007). Value at Risk and Bank Capital Management, Elsevier, Cambridge, MA.

Sinkala, W. and Nkalashe, T. (2015). Lie symmetry analysis of a first-order feedback model of option pricing, Advances in Mathematical Physics 2015, Article ID: 361785, DOI: $10.1155 / 2015 / 361785$

Takacs, L. (1995). On the local time of the Brownian motion, The Annals of Applied Probability 5(3): 741-756.

Vasicek, O. (1977). An equilibrium characterization of the term structure, Journal of Financial Economics 5(2): 177-188.

Michele Bonollo was born in Padova, Italy, in 1966. He received his $\mathrm{PhD}$ in statistics in 1996 at the University of Padova. He also holds a DEA (Msc degree) in stochastic models for finance from Paris VII. He denotes his time both to professional activities as well as academic applied research and teaching. He has worked for some of the most important Italian banks and advisory companies, mainly developing software systems for finance and risk management. Currently, he works at Numerix LLC, a leading company in quantitative finance solutions. His research spans from derivatives pricing to risk management with the related probabilistic and computational issues, as well as data quality and software design. He has published about 20 papers and has served as a speaker at dozens of scientific and professional conferences.

Luca Di Persio was born in Rome, Italy, in 1972. He received his $\mathrm{PhD}$ degree in mathematics from the University of Trento, along with the title of Doct. Rer. Naturalium from the University of Bonn in 2007. He is an assistant professor at the University of Verona, College of Mathematics, where he is the head of the Probability and Applications Group. His research interests span from the theory of stochastic processes to SPDEs, the study of interacting particle systems and neural networks architectures, and mathematical finance. All the aforementioned research topics have been developed in strict connection with a wide net of scientific collaborators all around the world as well as with several industries.
Luca Mammi received a BSc degree in physics from the University of Padova in 2008 and an MSc degree in finance from the CUOA Business School in 2012. Since 2012 he has worked as an analyst, focusing on credit risk modeling. From 2012 to 2013 he worked as an analyst at the Banco Popolare Banking Group, during the approval of the advanced internal rating based models and the following credit RWA optimization Since 2013 he has worked at the UniCredit Group, developing and validating credit VaR risk models and credit rating models.

Immacolata Oliva was born in Potenza, Italy, in 1982. She received her PhD in mathematics in 2012 from the University of Bologna. Currently, she is a post-doc fellow and an associate professor at the University of Verona, Department of Economics. Her research interests include the theory of stochastic processes and SPDEs with application to finance, stochastic control problems under partial information, and numerical methods applied to finance.

Received: 30 November 2016

Revised: 13 March 2017

Accepted: 4 February 2017 